\title{
ON THE DESIGN OF AN INNOVATIVE LATCH MECHANISM IN SMIFED WAFER CONTAINERS
}

\author{
Wei-Ming Pai $^{* *} \quad$ Dar-Zen Chen $^{*} \quad$ Jyh-Jone Lee $^{* *} \quad$ Chi-Zer Ho ${ }^{* *}$ \\ Department of Mechanical Engineering \\ National Taiwan University \\ Taipei, Taiwan 10617, R.O.C.
}

\begin{abstract}
This paper presents the design process for an innovative latch mechanism in a standard mechanical interfaced (SMIFed) wafer container, in which the manufactured integrated circuits are stored. An innovative latch mechanism is proposed and applied to the wafer container, such that the container door can be latched and air-tightly sealed during storage or transportation. The design process is divided into two stages. In the first stage, an output slot-cam is designed in order to generate decoupled fine motions of the output link. The issue is formulated as an optimization problem where the output link dimensions are optimized to minimize the resultant pin forces subject to an adequate transmission angle. In the second stage, the input slot-cam is designed to achieve that kinetic energy of the elastic gasket on the container lid is absorbed at a uniform rate. Finally, a numerical example and computer simulations are given to demonstrate the results of design process. It is believed that this work could aid in enhancing the performance and reliability of the latch mechanism in the SMIF environment.
\end{abstract}

Keywords : SMIF, Wafer container, Latch mechanism.

\section{INTRODUCTION}

The request for cleanliness in a semiconductorprocessing tool is unceasingly increased due to the enhancement of integrated circuit's (IC) integration. To ensure wafers against contamination, standard mechanical interfaced (SMIFed) environment [1 4] is constructed to interface a wafer container with the semiconductor-processing tool. Figure 1(a) shows a typical SMIFed mini-environment in IC manufacturing factories. The wafer container is used to create a particle-free and airtight mini-environment such that wafers can be prevented from contamination by abraded particles during transportation or storage. While operating, the wafer container is first mated to the port of processing equipment. An actuating device from outset is then engaged with the container door to activate the door latch mechanism, thereby releasing the container door from the wafer container. The latch mechanism inside the container door (see Fig. 1b) is utilized to secure the door with the wafer container and further seal the door to improve the air-tightness of the mini-environment. Various designs of latch mechanisms can be found in the literature [5 17], and are applied in different sizes of wafer containers. Since the latch mechanism is to be installed in a very space-limited environment inside the container door, the motions of the mechanism are crucial to the performance of the wafer container not only from the kinematic viewpoint but also from the wear viewpoint. Therefore, a comprehensive design methodology should take into account the issues as link dimensions, joint forces and characteristics of kinematic pairs. Moreover, such a design methodology should also accommodate the effect of the elastic gasket, placed on the door rim as a seal element, on the mechanism.

In a recent study [18], various innovative designs of latch mechanisms were conceived. In the mechanism design, it can be seen that the use of cam provides a relatively direct method to control mechanism motions [19 25]. An optimal design with output and input slot-cams is proposed and shown in Fig. 1(b). This mechanism consists of least link members and is able to present two decoupled latching functions, that is, a latch motion to secure the container door and a pressand-deform motion on the container case to improve the air-tightness of the container.

In this paper, we present a two-stage design procedure for the fine motions of the mechanism. Specifically, the output and input slot-cams are designed according to different requirements in the different two stages. In the first stage, the output slot-cam is first designed according to the motion requirements of the output link. Followed by that procedure, link

\footnotetext{
* Professor $\quad * *$ Associate Professor
} 


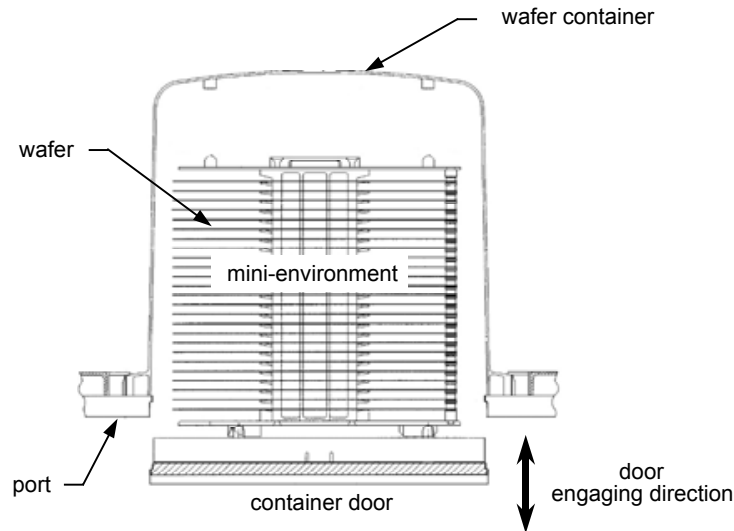

(a) Mini-environment
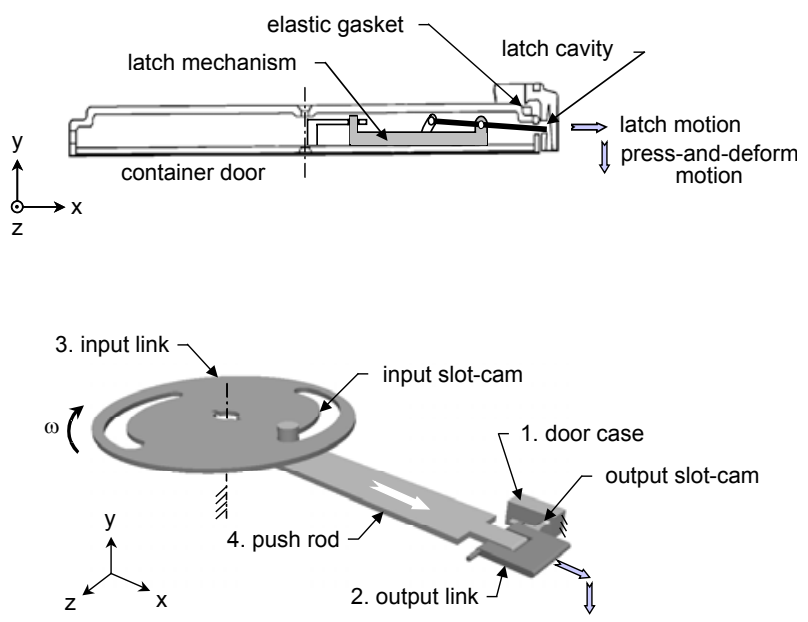

(b) An innovative latch mechanism

Fig. 1 Standard mechanical interface technique

dimensions are optimized by minimizing the joint forces on the output link, subject to an adequate transmission angle of the output slot-cam. In the second stage, the input slot-cam profile on the input link is determined based on the results of the first stage and divided by two parts. The front-end part is determined to let the output link move under a constant velocity. The back-end part is determined according to a decelerated deformation of the elastic gasket. This bases on the assumption of storing the kinetic energy of the deformed elastic gasket at a uniform rate. Finally, a numerical example and computer simulations are given to illustrate and verify the design results.

\section{MECHANISM DESCRIPTION}

As shown in Fig. 1(b), the latch mechanism is composed of four primary members: the output link (link 2), the input link (link 3), the push rod (link 4) and the door case (link 1). For convenience, an $x-y-z$ reference coordinate system is shown in Fig. 1(b). The vertical direction $(y)$ is defined as the door engaging direction, while the transverse direction $(x)$ is defined as the latching direction. The input link is pin-jointed to the door case and driven by a rotary actuator to rotate about $y$-axis. The input link drives the push rod to move along $x$-axis through an input slot-cam. The output link with its one end pin-jointed to the push rod and the other end guided by an output slot-cam slides towards the latch cavity. The tip of the output link then inserts into the latch cavity to secure the door with the wafer container and subsequently presses down on the rim of latch cavity. As a result, the elastic gasket underneath the container lid is deformed and the container door is air-tightly sealed with the door case.

In case a coupled motion exists in the mechanism during the latch and press-and-deform procedure, the output link will touch the latch cavity rim in a way of "gliding," resulting in abrasions between the link surfaces. In the long run, this may generate abraded particles to contaminate the wafers. Therefore, the latch and press-and-deform motions are expected to be decoupled, that is, the latch motion is performed followed by the press-and-down motion as shown in Fig. 1(b). For the latch motion, the tip of the output link is assumed to move at a constant velocity. Furthermore, during the press-and-deform motion, the tip of the output link is required to deform the gasket such that the kinetic energy of the input actuator is transmitted at a uniform rate.

\section{FIRST STAGE: OPTIMIZATION OF LINK DIMENSIONS}

\subsection{Derivation of Output Slot-Cam Profile}

Figure 2(a) shows the planar schematic of the innovative latch mechanism. The output link is pinjointed to the push rod at pivot A. Along the output slot-cam, pivot $\mathrm{P}$ moves to lever the tip B of output link. Figure 2(b) shows the two stages of the output link motion. During the latch motion, the tip B is requested to move linearly from an initial position $B$ to position $\mathrm{B}^{\prime}$ through a distance $m$. Following the latch motion, the press-and-deform motion requires the tip $\mathrm{B}^{\prime}$ to move down linearly to $\mathrm{B}^{\prime \prime}$ by a distance $h$. Thus, the first stage of output slot-cam can be designated as a straight slot, guiding pivot $\mathrm{P}$ to move linearly to position $\mathrm{P}^{\prime}$. The second stage of output slot-cam can be determined as follows. The location of $\mathrm{P}^{\prime \prime}$, with the zero position at $\mathrm{P}^{\prime}$ can be written as:

$$
x=\left(L_{1}+L_{2}\right) \cdot(1-\cos \theta)
$$

$$
y=L_{1} \cdot \sin \theta
$$

where $L_{1}$ is the length between pivots $\mathrm{P}$ and $\mathrm{A}, L_{2}$ the length between pivot $\mathrm{A}$ and tip $\mathrm{B}$, and $\theta$ the angle between the output link and $x$-axis.

For a specific downward displacement of $\mathrm{B}, h$, the maximum tilting angle of output link is

$$
\theta_{\max }=\sin ^{-1}\left(h / L_{2}\right)
$$




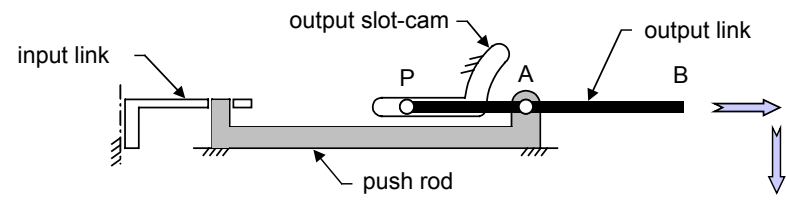

(a) Planar schematic of the innovative latch mechanism
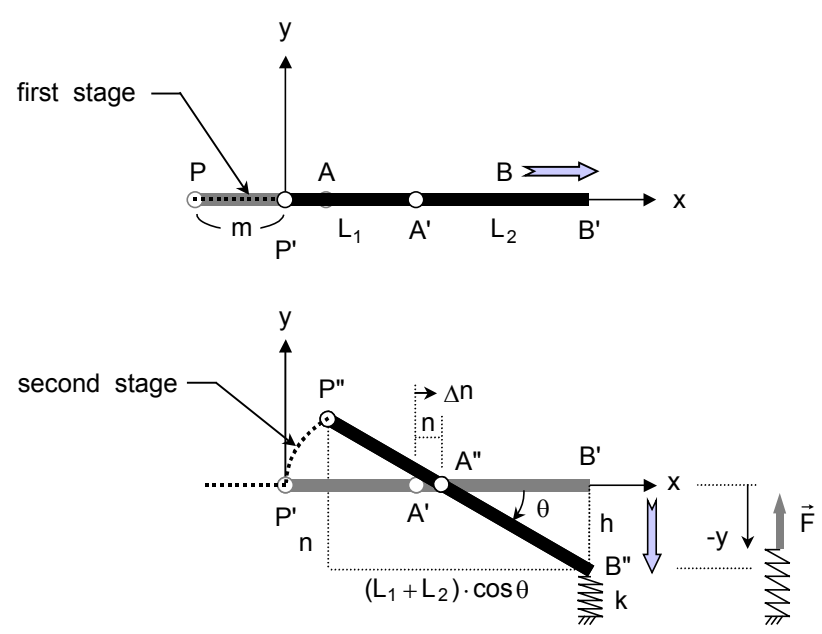

(b) Two stages of output slot-cam

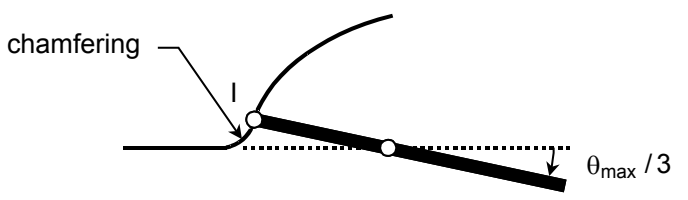

(c) Chamfering of the connection

Fig. 2 Derivation of output slot-cam profile

Thus, the locus of pivot $\mathrm{P}$ at the second stage $\left(\mathrm{P}^{\prime}\right.$ to $\left.\mathrm{P}^{\prime \prime}\right)$ can be obtained in terms of the design parameters $L_{1}$, $L_{2}$ and $h$.

The overall profile of output slot-cam is formed of the first and second slots mentioned above. However, if these two curves are jointed without any modification, there will exist an abruption in slope at the point of connection. In order to avoid this abruption, the connection is chamfered as shown in Fig. 2(c) from an engineering viewpoint, to yield a smooth motion of output link. Practically, the range of the chamfering $\theta$ can be distributed from zero to $\theta_{\max } / 3$.

\subsection{Optimization of Link Dimensions}

It can be seen that the ratio of link lengths $L_{1}$ and $L_{2}$ will affect the forces at joint $A$ and contact $P$. Since the mechanism is to be installed in a space-limited and ultra-clean environment, joint forces are crucial to the life cycle of the product. The less the joint forces are, the longer endurance and less abrasion will the mechanism parts possess. In what follows, the lengths $L_{1}$ and $L_{2}$ are determined by minimizing the resultant pin forces on the output link, subject to a constraint of an adequate transmission angle along the output slot-cam.

\section{Objective function}

As shown in Fig. 2(b), the elastic gasket can be modeled as a linear spring with a coefficient of elasticity $k$. The force $\vec{F}$, caused by deforming the spring of displacement $h$ can be written as

$$
\vec{F}=k \cdot h \cdot \vec{j}
$$

Figure 3(a) shows the free-body force diagram of the output link, in which $\overrightarrow{F_{P}}$ and $\overrightarrow{F_{A}}$ represent the resultand pin forces on pivots $\mathrm{P}^{\prime \prime}$ and $\mathrm{A}^{\prime \prime}$, respectively.

Writing the moment balance equation about $\mathrm{A}^{\prime \prime}$ yields,

$$
L_{1} \cdot \sin \theta \cdot F_{P x}+L_{1} \cdot \cos \theta \cdot F_{P y}-k \cdot h \cdot L_{2} \cdot \cos \theta=0
$$

where $F_{P x}$ and $F_{P y}$ are respectively the $x$ - and $y$-components of $\overrightarrow{F_{P}}$.

To solve for the unknown forces in Eq. (4), the ratio of the force components $F_{P x}$ and $F_{P y}$ can be obtained from the slope of the output slot-cam profile, since $\overrightarrow{F_{P}}$ is directing along the contact normal. The slope of $\overrightarrow{F_{P}}$ can be written as

$$
\frac{F_{P y}}{F_{P x}}=-\frac{d x}{d y}=-\frac{d x / d \theta}{d y / d \theta}=-\frac{\left(L_{1}+L_{2}\right) \cdot \sin \theta}{L_{1} \cdot \cos \theta}
$$

Substituting Eq. (5) into Eq. (4) yields

$$
\begin{gathered}
F_{P x}=-\frac{\cos \theta}{\sin \theta} \cdot k \cdot h \\
F_{P y}=\left(1+\frac{L_{2}}{L_{1}}\right) \cdot k \cdot h
\end{gathered}
$$

and

$$
\left|\overrightarrow{F_{P}}\right|=\sqrt{\left(\frac{\cos \theta}{\sin \theta}\right)^{2}+\left(\frac{L_{2}}{L_{1}}+1\right)^{2}} \cdot k \cdot h
$$

Writing the force balance equation obtains

$$
\overrightarrow{F_{P}}+\overrightarrow{F_{A}}+\vec{F}=0
$$

and

$$
\left|\overrightarrow{F_{A}}\right|=\sqrt{\left(\frac{\cos \theta}{\sin \theta}\right)^{2}+\left(\frac{L_{2}}{L_{1}}+2\right)^{2}} \cdot k \cdot h
$$

Thus, a design objective $Z$ is defined as the minimum of the sum of $\left|\overrightarrow{F_{P}}\right|$ and $\left|\overrightarrow{F_{A}}\right|$. 


$$
\begin{aligned}
Z: \min \left\{\left(\sqrt{\left(\frac{\cos \theta}{\sin \theta}\right)^{2}+\left(\frac{L_{2}}{L_{1}}+1\right)^{2}}\right.\right. \\
\left.\left.+\sqrt{\left(\frac{\cos \theta}{\sin \theta}\right)^{2}+\left(\frac{L_{2}}{L_{1}}+2\right)^{2}}\right) \cdot k \cdot h\right\}
\end{aligned}
$$

\section{$\underline{\text { Constraint function }}$}

In the design of latch mechanism, the transmission angle along the second stage of output slot-cam is related to the force transmission of the mechanism. Inadequate transmission angle would result in ineffective force transmission and approach to singular state. Hence, the transmission angle should be adequate, such that smooth motions of mechanism can be achieved. Figure 3(a) shows the transmission angle $\zeta$, the included angle between $\overrightarrow{F_{P}}$ and the direction of the output link.

$$
\begin{aligned}
\zeta & =\frac{\pi}{2}-\beta=\frac{\pi}{2}-\cos ^{-1}\left(\vec{V}_{1} \cdot \vec{V}_{2} /\left|V_{1}\right| \cdot\left|V_{2}\right|\right) \\
& =\frac{\pi}{2}-\cos ^{-1}\left(\frac{\sin \theta \cdot \cos \theta}{\sqrt{\sin ^{2} \theta+2 \cdot\left(\frac{L_{1}}{L_{2}}\right) \cdot \sin ^{2} \theta+\left(\frac{L_{1}}{L_{2}}\right)^{2}}}\right)
\end{aligned}
$$

where $\beta$ is the angle between the contact tangent $\vec{V}_{1}=\left(L_{1} \cdot \sin \theta+L_{2} \cdot \sin \theta, \quad L_{1} \cdot \cos \theta\right)$ and the direction of the output link $\overrightarrow{V_{2}}=(\cos \theta,-\sin \theta)$.

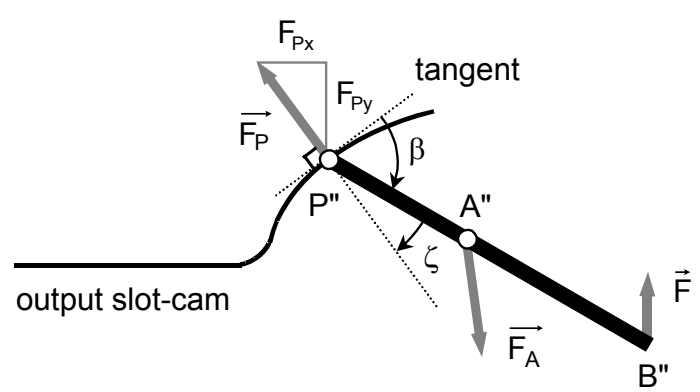

(a) Cam profile and free-body force diagram of output link

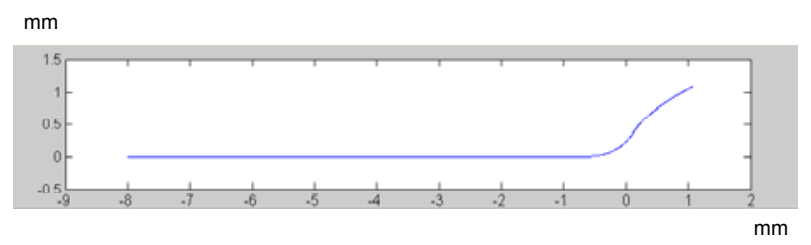

(b) Illustrative example

Fig. 3 Output slot-cam
From the numerical analysis, it can be seen that the transmission angle is increased while $\theta$ is increased for arbitrary $L_{1} / L_{2}$. Thus, the minimum value of transmission angle $\zeta_{\min }$ occurs at the initial point of the second stage of output slot-cam, namely point I, the end position of chamfering as shown in Fig. 2(c). Assuming $\zeta_{\min }$ is no less than a given value $\gamma$, the design constraint can be expressed as

$$
\zeta_{\min }=\zeta\left(\theta_{\max } / 3\right) \geq \gamma
$$

where $\theta_{\max }=\sin ^{-1}\left(h / L_{2}\right)$ as Eq. (2).

The lengths $L_{1}$ and $L_{2}$ can be obtained by solving the objective function Eq. (11) subject to the constraint function Eq. (13). Accordingly, profile of output slot-cam can be determined.

\subsection{Numerical Example}

A practical design was carried out based on the following specifications: the coefficient of elasticity $k=$ $10 \mathrm{~N} / \mathrm{mm}$, the press-and-down displacement $h=5 \mathrm{~mm}$ and the minimum constraint of transmission angle $\gamma=$ $\pi / 4$. Also, let the angle of output link, $\theta$, tilt from $0^{\circ}$ to $20^{\circ}$. The optimization problem was computed by the Matlab-Optimization Toolbox software [26]. The minimum value of the objective function $Z$ can be computed as $468.9 \mathrm{~N}\left(\left|\overrightarrow{F_{P}}\right|=218.5 \mathrm{~N},\left|\overrightarrow{F_{A}}\right|=250.4 \mathrm{~N}\right)$, the corresponding $L_{1} / L_{2}=0.22$ and $\theta_{\max }=20^{\circ}$. The lengths $L_{1}$ and $L_{2}$ can be respectively obtained as $3.18 \mathrm{~mm}$ and $14.62 \mathrm{~mm}$ by substituting $\theta_{\max }=20^{\circ}$ into Eq. (2). The optimal output slot-cam profile is shown in Fig. 3(b).

\section{SECOND STAGE: DERIVATION OF INPUT SLOT-CAM PROFILE}

The input link is assumed to rotate with a constant angular velocity $\omega$ by a specific rotary actuator. As shown in Fig. 4(a), the input slot-cam controls the motion of push rod, which subsequently transmits motions to the output link. The profile of input slot-cam is divided as two sections, respectively dominating the velocities of latch and press-and-down motions. As can be seen from the previous section, the push rod moves a total distance $m$ during the latch motion and then a total distance $n$ during the press-and-deform motion. Let the latch motion be a constant velocity case, the radius of the first section of the slot can be written as

$$
R_{1}=R_{0}+\Delta m=R_{0}+V_{0} \cdot t \quad 0 \leq t \leq t_{1}
$$

where $R_{0}$ is the radius of the initial position (base circle), $V_{0}$ the constant velocity of the push rod and $t_{1}$ the duration of the latch motion.

Assume that, the elastic gasket will act as a linear spring and store kinetic energy when deformed from B' 


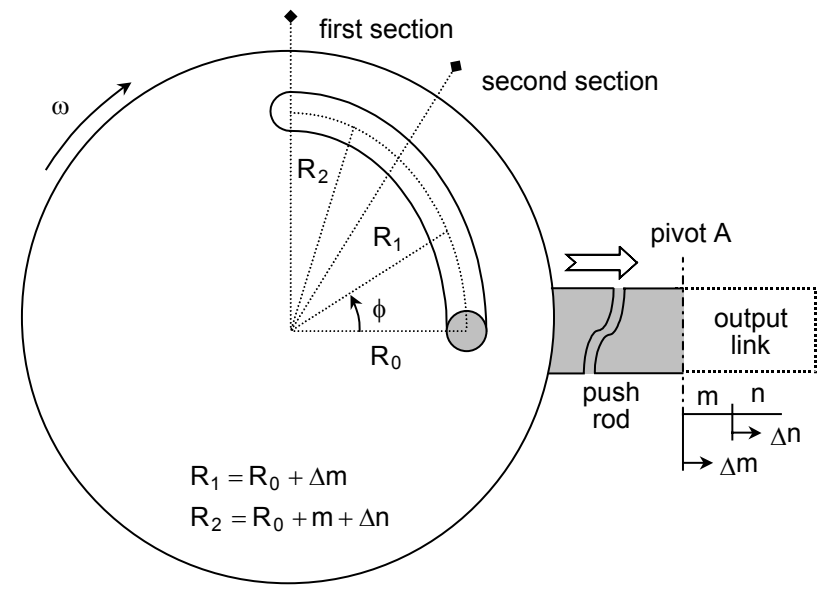

(a) Cam profile

$\mathrm{mm}$

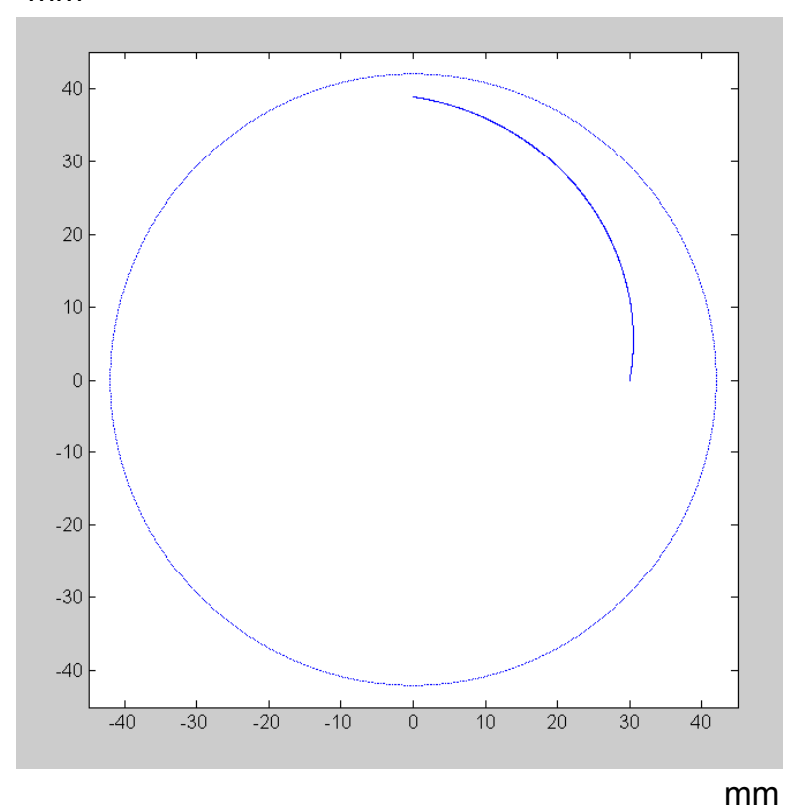

(b) Illustrative example

Fig. 4 Input slot-cam

to $\mathrm{B}^{\prime \prime}$ during the press-and-deform motion. Let the kinetic energy be stored at a constant rate.

$$
P=F \cdot \frac{d y}{d t}=k \cdot y \cdot \frac{d y}{d t}
$$

where $P$ is the storing rate of kinetic energy, $k$ the elasticity coefficient of the elastic gasket, $y$ the vertical displacement of the tip $\mathrm{B}^{\prime}$.

Solving Eq. (15) in terms of time $t$ obtains

$$
y=\sqrt{2 \frac{P}{k} t+2 \cdot C} \quad t_{1} \leq t \leq t_{2}
$$

where $C$ is a constant and $t_{2}$ is the total duration of whole motions.

From Fig. 2(b), horizontal shift of pivot $\mathrm{A}^{\prime}, \Delta n$, can be written as

$$
\begin{aligned}
\Delta n & =L_{2}-\sqrt{L_{2}{ }^{2}-y^{2}} \\
& =L_{2}-\sqrt{L_{2}{ }^{2}-2 \frac{P}{k} t-2 \cdot C} \quad t_{1} \leq t \leq t_{2}
\end{aligned}
$$

The radius of the second section of the slot can be thus expressed as

$$
\begin{aligned}
R_{2}= & R_{1}\left(t_{1}\right)+\Delta n=\left(R_{0}+m\right) \\
& +L_{2}-\sqrt{L_{2}{ }^{2}-2 \frac{P}{k} t-2 \cdot C} \quad t_{1} \leq t \leq t_{2}
\end{aligned}
$$

Equations (14) and (18) give the profile of the input slot-cam. The parameters $t_{1}, t_{2}, V_{0}, C$ and $P$ can be determined from the following boundary conditions.

(a) During the first section, the increase of radius equals to the displacement of latch motion, $m$.

$$
R_{1}\left(t_{1}\right)-R_{1}(0)=m
$$

(b) At the initial and final positions of the second section, the displacement of tip B in direction $y$ is of zero and $h$, respectively.

$$
\begin{aligned}
& y\left(t_{1}\right)=0 \\
& y\left(t_{2}\right)=h
\end{aligned}
$$

(c) For the purpose of continuity of the push rod motion, the ending velocity of the first section should be equal to the beginning velocity of the second section.

$$
\dot{R}_{1}\left(t_{1}\right)=\dot{R}_{2}\left(t_{1}\right)
$$

With a specific angular displacement $\alpha$ and a constant angular velocity $\omega$ of the actuator, the duration $t_{2}$ can be obtained as $\alpha / \omega$. Solving Eqs. (19), (20) and (21) obtains

$$
\begin{gathered}
t_{1}=\frac{2 \cdot \alpha \cdot m \cdot L_{2}}{\omega \cdot\left(h^{2}+2 \cdot m \cdot L_{2}\right)} \\
V_{0}=\frac{\omega \cdot\left(h^{2}+2 \cdot m \cdot L_{2}\right)}{2 \cdot \alpha \cdot L_{2}} \\
C=-m \cdot L_{2}
\end{gathered}
$$

and

$$
P=\frac{k \cdot \omega \cdot m \cdot L_{2}}{\alpha}
$$

For the numerical example, the parameters are chosen as follows: $\alpha=\pi / 2, \omega=63 \mathrm{rpm}, k=10 \mathrm{~N} / \mathrm{mm}, h$ $=5 \mathrm{~mm}, m=8 \mathrm{~mm}, L_{2}=14.62 \mathrm{~mm}$ and $R_{0}=30 \mathrm{~mm}$. Corresponding values of $t_{1}=0.215 \mathrm{~s}, t_{2}=0.238 \mathrm{~s}, V_{0}=$ $37.2 \mathrm{~mm} / \mathrm{s}, \mathrm{C}=-116.96$ and $\mathrm{P}=5.44 \mathrm{Nm} / \mathrm{s}$. Figure $4(\mathrm{~b})$ shows the input slot-cam profile of the illustrative example. 


\section{COMPUTER SIMULATIONS}

Based on the optimized results and corresponding output and input slot-cam profiles in Figs. 3(b) and 4(b), the solid model of the latch mechanism is built as shown in Fig. 1(b). The mechanism motions are simulated by software Working Model for demonstration. A real prototype is also constructed and verified. Figure 5(a) shows the simulated motions of the output link and the path of the tip B. It can be seen that the profile of output slot-cam effectively guides the tip B to move transversely and then vertically. The decoupled latch and press-and-deform motions are therefore performed. Note that the curved connection between the two motions is caused by the chamfering of the output slot-cam profile. Figure 5(b) indicates the displacements of latch motion as well as the press-anddeform motion. Figure 5(c) also shows the velocity of tip B of output link. The output link moves at a constant speed $37 \mathrm{~mm} / \mathrm{s}$ during the latch motion $\left(V_{x}\right)$. Due to chamfering effect on the output slot-cam, the speed then diminishes to zero gradually. Following the latch motion, the output link starts to move downward and then decelerates $\left(V_{y}\right)$. The trend of velocity conforms to our design expectation. Note that the shoots of the velocity curve indicate the collisions between links, since a tolerance of $0.005 \mathrm{~mm}$ is taken into consideration during the simulation.

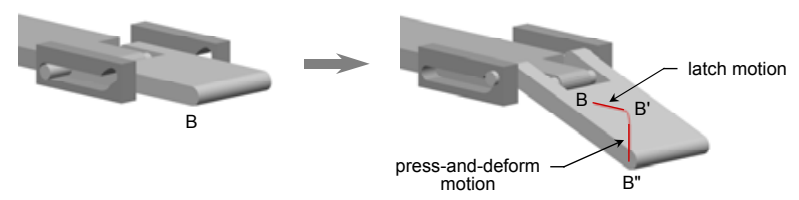

(a) latch and press-and-deform motions

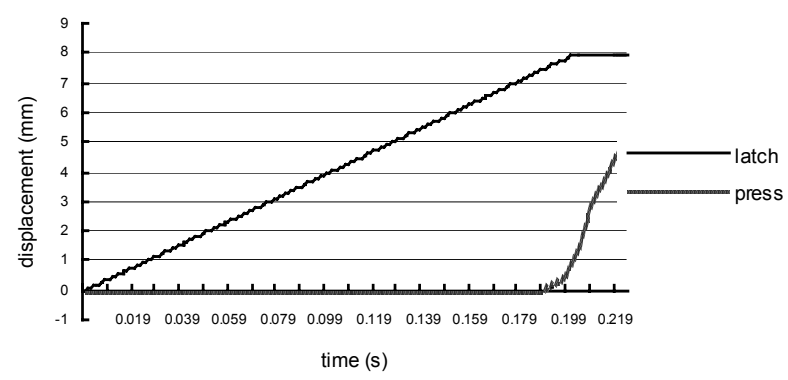

(b) displacement diagram

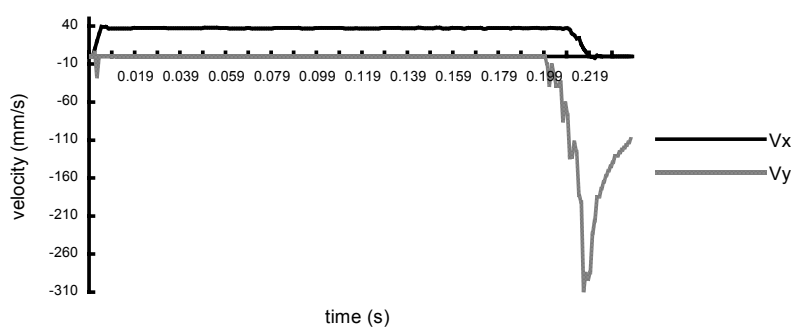

(b) velocity diagram

Fig. 5 Simulation results

\section{CONCLUSION}

In this paper, the design of an innovative latch mechanism is presented. The output slot-cam is first conceived to perform the motion requirements of the wafer container product. The link dimensions are then determined to minimize the resultant pin forces in the mechanism. The input slot-cam profile is finally determined by taking account of the speed requirements for the output link. Through computer simulations, the design results are also verified. Since decoupled output motions and proper motion velocities are yielded, this design potentially increases the endurance of the mechanism and reduces the abrasions between mechanism members.

\section{ACKNOWLEDGMENTS}

The financial support of the National Science Council (NSC-92-2212-E-002-039) is gratefully appreciated.

\section{REFERENCES}

1. Parikh, M., Kaempf, U., "SMIF: A Technology for Wafer Cassette Transfer in VLSI Manufacturing," Solid State Technology, Vol. 27, No. 7, pp. 111-115 (1984).

2. Doche, C., "Wafer Confinement for Control of Contamination in Microelectronics," Solid State Technology, Vol. 33, No. 8, pp. S1-S5 (1990).

3. Book of SEMI Standards, SEMI E19-0697.

4. Book of SEMI Standards, SEMI E62-0999.

5. United States Patent No. 4,674,939.

6. United States Patent No. 4,995,430.

7. United States Patent No. 5,586,585.

8. United States Patent No. 5,607,276.

9. United States Patent No. 5,609,459.

10. United States Patent No. 5,613,821.

11. United States Patent No. 5,711,427.

12. United States Patent No. 5,740,845.

13. United States Patent No. 5,743,424.

14. United States Patent No. 5,752,796.

15. United States Patent No. 5,931,512.

16. United States Patent No. 5,957,292.

17. United States Patent No. 5,988,392.

18. Pai, Wei-Ming, Chen, Dar-Zen, Lee, Jyh-Jone and Wu, Tzong-Ming, "A Decomposition Methodology for Design of Mechanisms from Functional and Structural Perspectives," Journal of the Chinese Institute of Engineers, Vol. 26, No. 4, pp. 537-543 (2003).

19. Ananthasuresh, G. K., "Design of Fully Rotatable, Roller-Crank-Driven, Cam Mechanisms for 
Arbitrary Motion Specifications," Mechanism and Machine Theory, Vol. 36, No. 4, pp. 445-467 (2001).

20. Kota, S., and Erdman, A. G., "Motion Control in Product Design," Mechanical Engineering, Vol. 119, No. 8, pp. 74-77 (1997).

21. Liaw, D. G., "Synthesis of Cam-Link Mechanisms for Exact Position Guidance of Rigid Bodies," Journal of the Chinese Society of Mechanical Engineers, Vol. 5, No. 1, pp. 27-43 (1984).

22. Mills, J. K., Notash, L. and Fenton, R. G., "Optimal Design and Sensitivity Analysis of Flexible Cam Mechanisms," Mechanism \& Machine Theory, Vol. 28, No. 4, pp. 563-581 (1993).

23. Yu, Q., and Lee, H. P., "Size Optimization of Cam Mechanisms with Translating Roller Followers," Proceedings of the Institution of Mechanical Engineers, Part C: Journal of Mechanical
Engineering Science, Vol. 212, No. 5, pp. 381-386 (1998).

24. Bouzakis, K. D., Mitsi, S. and Tsiafis, J., "Computer-aided Optimum Design and NC Milling of Planar Cam Mechanisms," International Journal of Machine Tools \& Manufacture, Vol. 37, No. 8, pp. 1131-1142 (1997).

25. Sadler, J. P. and Yang, Zhijia, "Optimal Design of Cam-Linkage Mechanisms for Dynamic-Force Characteristics," Mechanism \& Machine Theory, Vol. 25, No. 1, pp. 41-57 (1990).

26. MATLAB, The Language of Technical Computing, Version 6.0.0.88, Release 12 (2000).

(Manuscript received Jan. 13, 2002, Accepted for publication May 30, 2003.) 